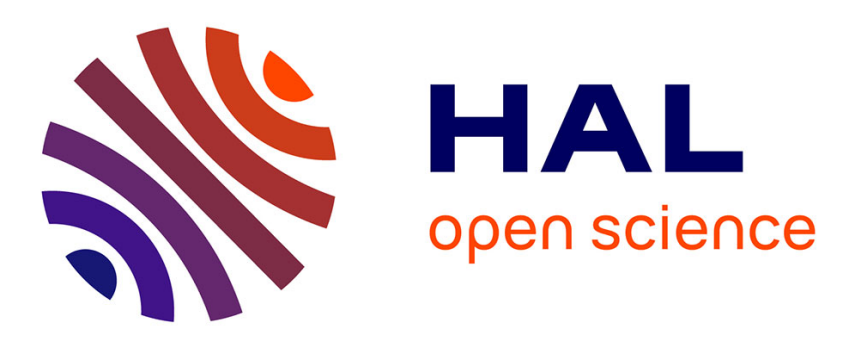

\title{
A modified two-steps LMI method to design observer-based controller for linear discrete-time systems with parameter uncertainties
}

Cherifa Bennani, Fazia Bedouhene, Ali Zemouche, Hamza Bibi, Abdel Aitouche

\section{To cite this version:}

Cherifa Bennani, Fazia Bedouhene, Ali Zemouche, Hamza Bibi, Abdel Aitouche. A modified twosteps LMI method to design observer-based controller for linear discrete-time systems with parameter uncertainties. 6th International Conference on Systems and Control, ICSC 2017, May 2017, Batna, Algeria. 10.1109/icosc.2017.7958736 . hal-01567355

\section{HAL Id: hal-01567355 \\ https://hal.science/hal-01567355}

Submitted on 12 Jan 2018

HAL is a multi-disciplinary open access archive for the deposit and dissemination of scientific research documents, whether they are published or not. The documents may come from teaching and research institutions in France or abroad, or from public or private research centers.
L'archive ouverte pluridisciplinaire $\mathbf{H A L}$, est destinée au dépôt et à la diffusion de documents scientifiques de niveau recherche, publiés ou non, émanant des établissements d'enseignement et de recherche français ou étrangers, des laboratoires publics ou privés. 


\title{
A Modified Two-Steps LMI Method to Design Observer-based controller for Linear Discrete-Time Systems with Parameter Uncertainties
}

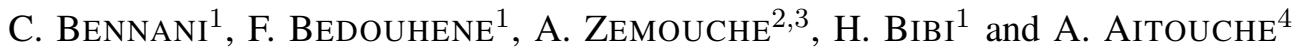

\begin{abstract}
This note deals with the problem of observerbased stabilization for discrete-time linear systems with normbounded parameter uncertainties. Thanks to slack variable technique and the two-step method, an LMI-based approach is provided to compute simultaneously all the main decision variable of the observer-based controller problem. Our approach is inspired from the classical two-steps method introduced by Stankovic et al. and the modified two-steps method introduced by Zemouche et al.. Some comments are reserved to emphasize and clarify the difference between the different variants of the two-steps method. A numerical example is provided in order to illustrate how the new algorithm is less conservative than previous results in literature.
\end{abstract}

Index Terms-Observer-based control; Linear matrix inequalities (LMIs); uncertain systems; two steps method.

\section{INTRODUCTION}

Over the last decades, given its importance, the ObserverBased Stabilization problem for systems with parameter uncertainties is constantly arousing the interest of the automation community, both in continuous and discrete-time cases. Several studies have been devoted to the issue, in the LMI framework, to offer partial solutions. To date, the problem is not completely solved because of its difficulty. In the discrete-time systems, one can mention, for example, the paper by Lien [1], concerned with the equality constraint method. Ibrir et al. have considered the same problem where, in order to linearize some bilinear term, the technique of constraint equality [2], the decoupling technique [3], and the technique of the stack variables [4], [5] are used. An other method which deals with the same issue is proposed by Kheloufi et al. [6], [7], and Zemouche et al. [8]. Their idea is to exploit judiciously the Young inequality. Contrarily to the contraint equality approach, the so-called Young's inequality-based approach, has the avantage that it works successfully independently of the dimension of the input, in addition, the necessary condition for the feasibility of proposed LMI is equivalent to the stabilizability and detectability of the system (without uncertainties) [6]. But it has the disadvantage that, for the solvability of the LMI condition, it requires an a priori choice of some scalar variable coming from the use of the Young inequality.

\footnotetext{
${ }^{1}$ Laboratoire de Mathématiques Pures et Appliques, University Mouloud Mammeri, Tizi-Ouzou, BP No 17 RP 15000, Algeria.

2 University of Lorraine, 186, rue de Lorraine, CRAN UMR CNRS 7039, 54400 Cosnes et Romain, France (email: ali.zemouche@ univ-lorraine.fr

${ }^{3}$ EPI Inria DISCO, Laboratoire des Signaux et Systèmes, CNRS-Centrale Supelec, 91192 Gif-sur-Yvette, France (email: ali.zemouche@ inria. fr

${ }^{*}$ CRIStAL Laboratory, HEI School, Lille, France.
}

Several papers in the literature have addressed the robust stabilization issue via constant gain Luenberger observers for linear systems with parametric uncertainties, but the results remain conservative. We refer the readers to [9], [10], for some interesting works in this area. In this paper, we address the problem of observer-based control design via LMIs. We propose new and enhanced LMI conditions to solve the problem of stabilization of linear systems with uncertain parameters. The proposed design methodology is related to the approach presented in [11]. Indeed, we propose a new variant of two-steps procedure, which contains, as particular case, that introduced by Zemouche et al. [11]. We show that this new variant encompasses the Young's relation based approach [7] and the two-steps methods [12], [11]. The rest of this paper is organized as follows: Section $[\Pi$ is devoted to the problem statement and a brief state of the art review related to different LMI design methods available in the literature to examine what already exists in the academic literature. The main contribution is presented and proved in Section III. A numerical example is added in Section IV] to demonstrate the validity and the effectiveness of the proposed methodology. Finally, we end the paper by a conclusion.

\section{PRoblem StATEMENT AND BACKGROUND RESUlts}

\section{A. Problem statement}

Consider the same class of systems investigated in [7]:

$$
\begin{gathered}
x_{t+1}=(A+\Delta A(t)) x_{t}+B u_{t} \\
y_{t}=(C+\Delta C(t)) x_{t}
\end{gathered}
$$

where $t=0,1, \ldots, x_{t} \in \mathbb{R}^{n}$ is the state vector, $y_{t} \in \mathbb{R}^{p}$ is the output measurement, and $u_{t} \in \mathbb{R}^{m}$ is the control input vector. The nominal matrices $A \in \mathbb{R}^{n \times n}, B \in \mathbb{R}^{n \times m}$, and $C \in \mathbb{R}^{p \times n}$ are known. The pairs $(A, B)$ and $(A, C)$ are assumed to be stabilizable and detectable, respectively. The uncertain terms $\Delta A(t) \in \mathbb{R}^{n \times n}$ and $\Delta C(t) \in \mathbb{R}^{p \times n}$ are unknown matrices that account for time-varying parameter uncertainties and are assumed to be of the form

$$
\Delta A(t)=M_{A} F_{A}(t) N_{A}, \quad \Delta C(t)=M_{C} F_{C}(t) N_{C}
$$

where $M_{A}, N_{A}, M_{C}$, and $N_{C}$ are known real constant matrices; $F_{A}(t)$ and $F_{C}(t)$ are unknown real time-varying matrices satisfying the inequalities

$$
F_{A}^{T}(t) F_{A}(t) \leq \alpha^{2} I, \quad F_{C}^{T}(t) F_{C}(t) \leq \beta^{2} I, \quad \forall t \in \mathbb{N} .
$$

For the sake of simplicity, from now on we will denote $\Delta A(t)$ and $\Delta C(t)$ by $\Delta A$ and $\Delta C$, respectively. Such a 
notation will be adopted also for all the matrices that depend on $\Delta A(t)$ and $\Delta C(t)$.

The asymptotic stabilization of the discrete-time linear system (1) is addressed using the Luenberger observer

$$
\hat{x}_{t+1}=A \hat{x}_{t}+B u_{t}+L\left(y_{t}-C \hat{x}_{t}\right)
$$

where $L \in \mathbb{R}^{n \times p}$ is the observer gain to be determined and $\hat{x}_{t} \in \mathbb{R}^{n}$ is the estimate of $x_{t}$ at time $t$. Let $e_{t}:=x_{t}-\hat{x}_{t}$ be the estimation error. Then, under the feedback

$$
u_{t}=-K \hat{x}_{t}
$$

where $K \in \mathbb{R}^{m \times n}$ is the controller gain, the closed-loop dynamics is described by

$$
z_{t+1}=\left[\begin{array}{cc}
A-B K+\Delta A & B K \\
\Delta A-L \Delta C & A-L C
\end{array}\right] z_{t}=\Pi z_{t}
$$

where $z_{t}^{T}=\left(x_{t}^{T}, e_{t}^{T}\right) \in \mathbb{R}^{2 n}$ is the augmented state vector.

The goal is to find suitable controller and observer gains $K$ and $L$ that guarantee the asymptotic stability of (6). Toward this end, let $V_{t}:=V\left(z_{t}\right)=z_{t}^{T} P z_{t}$ with $P>0$ be a Lyapunov function. The closed-loop system (6) is asymptotically stable if there exists a matrix $P>0$ such that the difference between the Lyapunov functions at two consecutive time instants $\Delta V_{t}:=V_{t+1}-V_{t}$ is negative definite, that is,

$$
\Delta V_{t}=z_{t}^{T}\left(\Pi^{T} P \Pi-P\right) z_{t}<0, \forall z_{t} \neq 0, \forall t \in \mathbb{N}
$$

Using Schur Lemma, (7) turns out to be equivalent to:

$$
\left[\begin{array}{cc}
-P & \Pi \\
(\star) & -P^{-1}
\end{array}\right]<0
$$

\section{B. Background results}

Here, we will recall some LMI methods concerned with this note. First, we recall the so-called Young's inequalitybased approach established in [7] for asymptotic stability of the system (6). After this, we summarize the LMI design established in [3]. The end of this section will be devoted to a useful linearization Lemma [3] and [4], which gives a sufficiency condition for linearizing some BMI. We announce it and give the necessary condition part.

Theorem 1 ([7]): If there exist $\epsilon_{2}>0$, two symmetric positive definite matrices $\mathcal{P}=\left[\begin{array}{ll}\mathcal{P}_{11} & \mathcal{P}_{12} \\ \mathcal{P}_{12}^{T} & \mathcal{P}_{22}\end{array}\right] \in \mathbb{R}^{2 n \times 2 n}$ and $\mathcal{G}_{1} \in \mathbb{R}^{n \times n}, G_{2} \in \mathbb{R}^{n \times n}$ invertible, $\hat{K} \in \mathbb{R}^{m \times n}$, and $\hat{L} \in$ $\mathbb{R}^{n \times p}$ such that the LMI

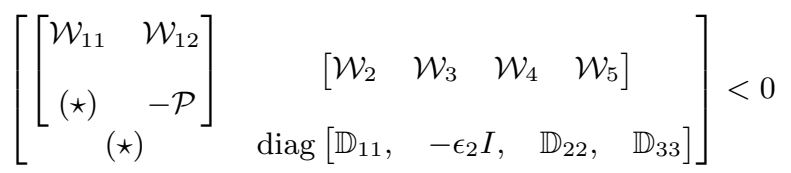

with

$$
\begin{aligned}
& \mathcal{W}_{11}=\left[\begin{array}{cc}
\mathcal{P}_{11}-2 \mathcal{G}_{1}+\epsilon_{2} M_{A} M_{A}^{T} & \mathcal{P}_{12} \\
\mathcal{P}_{12}^{T} & \mathcal{P}_{22}-\left(G_{2}+G_{2}^{T}\right)
\end{array}\right] \\
& \mathcal{W}_{12}=\operatorname{diag}\left[A \mathcal{G}_{1}-B \hat{K},\right. \\
& \mathcal{W}_{2}=\left[\begin{array}{cccc}
\hat{K}^{T} B^{T} & 0 & 0 & 0 \\
0 & 0 & 0 & I
\end{array}\right]^{T}, \mathbb{D}_{11}=\operatorname{diag}\left[\begin{array}{ll}
-\epsilon_{1} & -\epsilon_{1}^{-1}
\end{array}\right] \mathcal{G}_{1} \\
& \mathcal{W}_{3}=\left[\begin{array}{llll}
0 & 0 & N_{A} \mathcal{G}_{1} & 0
\end{array}\right]^{T}, \mathbb{D}_{22}=\operatorname{diag}\left[\begin{array}{ll}
-\epsilon_{3} & -\epsilon_{3}^{-1}
\end{array}\right] I \\
& \mathcal{W}_{4}=\left[\begin{array}{cccc}
0 & M_{A}^{T} G_{2} & 0 & 0 \\
0 & 0 & N_{A} \mathcal{G}_{1} & 0
\end{array}\right]^{T}, \mathbb{D}_{33}=\operatorname{diag}\left[-\epsilon_{4}, \frac{-1}{\epsilon_{4}}\right] I \\
& \mathcal{W}_{5}=\left[\begin{array}{cccc}
0 & M_{C}^{T} \hat{L}^{T} & 0 & 0 \\
0 & 0 & N_{C} \mathcal{G}_{1} & 0
\end{array}\right]^{T}
\end{aligned}
$$

is feasible for some positive constants $\epsilon_{1}, \epsilon_{3}$, and $\epsilon_{4}$, then (6) is asymptotically stable with $K=\hat{K} \mathcal{G}_{1}^{-1}$ and $L=$ $\left(G_{2}^{T}\right)^{-1} \hat{L}$.

The proof of Theorem 1 is based on the slack variables technique combined with the judicious handling of the famous Young's inequality.

Theorem 2 ([3]): Consider system (1) and observer (4). If there exist two symmetric and positive definite matrices $P_{1} \in \mathbb{R}^{n \times n}, P_{2} \in \mathbb{R}^{n \times n}$, two real matrices $Y_{1} \in \mathbb{R}^{m \times n}, Y_{2} \in \mathbb{R}^{n \times p}$, and five positive constants $\alpha, \beta, \epsilon_{1}, \epsilon_{2}, \epsilon_{3}$ such that the following holds:

$$
\begin{aligned}
& {\left[\begin{array}{cc}
P_{1} & I \\
\star & (2 \beta-\alpha) I
\end{array}\right]>0,} \\
& {\left[\begin{array}{cccccc}
\sigma_{11} & A P_{1}+B Y_{1} & B Y_{1} & 0 & 0 & 0 \\
\star & -P_{1} & 0 & P_{1} N_{A}^{T} & P_{1} N_{C}^{T} & P_{1} N_{A}^{T} \\
\star & \star & -\alpha I & 0 & 0 & 0 \\
\star & \star & \star & -\epsilon_{1} I & 0 & 0 \\
\star & \star & \star & \star & -\epsilon_{2} I & 0 \\
\star & \star & \star & \star & \star & -\epsilon_{3} I
\end{array}\right]<0} \\
& {\left[\begin{array}{ccccc}
-P_{2} & \Gamma_{12} & \beta I & 0 & 0 \\
\star & -P_{2} & 0 & P_{2} M_{A} & Y_{2} M_{C} \\
\star & \star & -P_{1} & 0 & 0 \\
\star & \star & \star & -\left(2-\epsilon_{2}\right) I & 0 \\
\star & \star & \star & \star & -\left(2-\epsilon_{3}\right) I
\end{array}\right]<0}
\end{aligned}
$$

where $\sigma_{11}=-P_{1}+\epsilon_{3} M_{A} M_{A}^{T}$ and $\Gamma_{12}=A^{T} P_{2}+C^{T} Y_{2}^{T}$, then there exist two gains $L=P_{2}^{-1} Y$ et $K=Y_{1} P_{1}^{-1}$ such that systems (1) and (4) are globally asymptotically stable under the feedback (5).

Beside some congruence transformation, one of the main ideas adopted in the proof of Theorem 2 is the following so-called Ibrir's Linearization Lemma, which proposes an interesting solution to the problem of coexistence of dependent variables in LMI framework, such as a variable and its inverse.

Lemma 1 ([4]): Given the matrices $X, Y$, and $Z$ of appropriate dimensions where $X=X^{\top}>0$ and $Z=Z^{\top}>$ 0 . Then, the following linear matrix inequality holds:

$$
\left[\begin{array}{cc}
-X & Y^{\top} \\
Y & -Z^{-1}
\end{array}\right]<0
$$

if there exists a positive constant $\alpha>0$ such that

$$
\left[\begin{array}{ccc}
-X & \alpha Y^{\top} & 0 \\
\alpha Y & -2 \alpha I & Z \\
0 & Z & -Z
\end{array}\right]<0
$$

This lemma proposed and proved by Ibrir [4] has also exploited in the nonlinear context. 
Remark 1: Let us mention that (13) and (14) are not equivalent. Indeed, we propose here an example of matrix $X, Y$ and $Z$ satisfying (13), and for which there is no $\alpha>0$ such that (14) holds. Take

$$
X=\left[\begin{array}{ll}
4 & 1 \\
1 & 6
\end{array}\right], Y^{T}=\left[\begin{array}{ll}
1 & 0 \\
2 & 0
\end{array}\right], Z=\left[\begin{array}{ll}
1 & 1 \\
1 & 2
\end{array}\right] .
$$

With this choice of $X=X^{\top}>0, Z=Z^{\top}>0$ and $Y$, inequality (13) is satisfied. Now, if we assume that there exists $\alpha>0$ such that 14 holds, then we get, using Sylvester's criterion, that all of its principal minors of odd order are negative. In particular, we have

$$
\Delta_{5}(\alpha)=2 \alpha\left(18 \alpha^{2}-55 \alpha+46\right)<0,
$$

which is impossible since $\Delta_{5}(\alpha)>0$ for all $\alpha>0$.

\section{New LMI Design Procedure}

In this section, we shall present a numerically efficient technique to find the observer and controller gains in such a way to stabilize system in closed loop, i.e., to ensure that (6) is asymptotically stable. In order to derive sufficiency conditions that guarantee the stability asymptotic of $(6)$, we begin by the step of linearization of the BMI (8). Here, instead of using Lemma 1 , we use the following well-known Theorem of De Oliveira et al. [13].

Theorem 3 ([13]): The following conditions are equivalent:

1) There exists a symmetric matrix $P>0$ such that

$$
A^{T} P A-P<0 \text {. }
$$

2) There exist a symmetric matrix $P$ and a matrix $G$ such that

$$
\left[\begin{array}{cc}
P & A^{T} G^{T} \\
G A & G+G^{T}-P
\end{array}\right]>0
$$

Using Theorem 3, the BMI problem (8) is equivalent to find an invertible matrix $G$ and a symmetric positive definite matrix $P$ such that

$$
\left[\begin{array}{cc}
P-\operatorname{He}(G) & G \Pi \\
(\star) & -P
\end{array}\right]<0,
$$

where $\operatorname{He}(G)=G+G^{T}$. Hence, after developing the product $G \Pi$ in (18), we obtain by choosing $G=\operatorname{diag}\left(G_{1}, G_{2}\right)$

$$
\left[\begin{array}{ccc}
P-\operatorname{He}(G) & {\left[\begin{array}{cc}
G_{1}(A-B K+\Delta A) & G_{1} B K \\
G_{2}(\Delta A-L \Delta C) & G_{2}(A-L C)
\end{array}\right]} \\
(\star) & -P &
\end{array}\right]<0 .
$$

Let at first deal with the linearization of the uncertainties in (19). We decompose the matrix defining (19) as follows:

$$
\begin{aligned}
& \Omega:=\left[\begin{array}{ccc}
P-\operatorname{He}(G) & {\left[\begin{array}{cc}
G_{1}(A-B K+\Delta A) & G_{1} B K \\
G_{2}(\Delta A-L \Delta C) & G_{2}(A-L C)
\end{array}\right]} \\
(\star) & -P &
\end{array}\right] \\
& =\overbrace{\left[\begin{array}{crr}
P-\operatorname{He}(G) \\
(\star)
\end{array}\left[\begin{array}{rr}
G_{1} A-\underbrace{}_{G_{1} B K} & \sigma^{G_{1} B K} \\
0 & G_{2}(A-L C)
\end{array}\right]\right.}^{\Omega_{1}}
\end{aligned}
$$

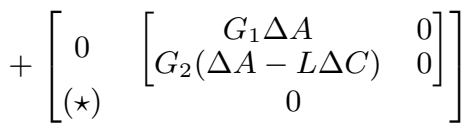

$$
\begin{aligned}
& =\Omega_{1}+\operatorname{He}\left\{\left[\begin{array}{c}
G_{1} M_{A} \\
G_{2} M_{A} \\
0 \\
0
\end{array}\right] F_{A}(t)\left[\begin{array}{c}
0 \\
0 \\
N_{A}^{T} \\
0
\end{array}\right]^{T}\right\} \\
& +\operatorname{He}\left\{\left[\begin{array}{c}
0 \\
G_{2} L M_{C} \\
0 \\
0
\end{array}\right] F_{C}(t)\left[\begin{array}{c}
0 \\
0 \\
-N_{C}^{T} \\
0
\end{array}\right]^{T}\right\} .
\end{aligned}
$$

Now, by applying the classical Young's relation on the righthand side of inequality (20), we get for any $\epsilon_{1}>0$ and $\epsilon_{2}>0$ :

$$
\begin{gathered}
\Omega \leq \Omega_{1}+\frac{1}{\epsilon_{1}}\left[\begin{array}{c}
G_{1} M_{A} \\
G_{2} M_{A} \\
0 \\
0
\end{array}\right]\left[\begin{array}{c}
G_{1} M_{A} \\
G_{2} M_{A} \\
0 \\
0
\end{array}\right]^{T}+\alpha^{2} \epsilon_{1}\left[\begin{array}{c}
0 \\
0 \\
N_{A}^{T} \\
0
\end{array}\right]\left[\begin{array}{c}
0 \\
0 \\
N_{A}^{T} \\
0
\end{array}\right]^{T} \\
+\frac{1}{\epsilon_{2}}\left[\begin{array}{c}
0 \\
G_{2} L M_{C} \\
0 \\
0
\end{array}\right]\left[\begin{array}{c}
0 \\
G_{2} L M_{C} \\
0 \\
0
\end{array}\right]^{T}+\beta^{2} \epsilon_{2}\left[\begin{array}{c}
0 \\
0 \\
N_{C}^{T} \\
0
\end{array}\right]\left[\begin{array}{c}
0 \\
0 \\
N_{C}^{T} \\
0
\end{array}\right] .
\end{gathered}
$$

Therefore, from the estimate (21) and the changes of variables $\hat{L}=G_{2} L, \hat{\alpha}^{-1}=\epsilon_{1} \alpha^{2}$, and $\hat{\beta}^{-1}=\epsilon_{2} \beta^{2}$, we deduce that the inequality $(8)$ holds if

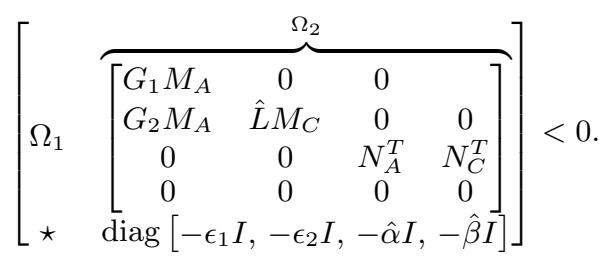

Now, it remains to linearize the bilinear term $G_{1} B K$ involved in (22). Since $B$ is full column rank, there always exists a non singular matrix $T$ so that $T B=\left[\begin{array}{c}I_{m} \\ 0\end{array}\right]$. Hence, using the similarity transformation $T$, system (1) can be transformed under the following equivalent form:

$$
\begin{gathered}
A:=T A T^{-1}, C:=C T^{-1}, B:=T B=\left[\begin{array}{c}
I_{m} \\
0
\end{array}\right], \\
\Delta A(t):=T \Delta A(t) T^{-1}, \Delta C(t):=\Delta C(t) T^{-1}, \\
M_{A}:=T M_{A}, N_{A}:=N_{A} T^{-1}, M_{C}:=M_{C}, N_{C}:=N_{C} T^{-1} .
\end{gathered}
$$

Consequently, in the rest of this note, we assume without loss of generality that

$$
B=\left[\begin{array}{c}
I_{m} \\
0
\end{array}\right] .
$$

Now, we need to write the matrix $G_{1}$ in the detailed forms:

$$
G_{1}=\left[\begin{array}{ll}
G_{11}^{1} & G_{12}^{1} \\
G_{21}^{1} & G_{22}^{1}
\end{array}\right] .
$$

Taking into account the structure 23) of $B$, we have:

$$
G_{1} B=\left[\begin{array}{ll}
G_{11}^{1} & G_{12}^{1} \\
G_{21}^{1} & G_{22}^{1}
\end{array}\right]\left[\begin{array}{c}
I_{m} \\
0
\end{array}\right]=\left[\begin{array}{l}
G_{11}^{1} \\
G_{21}^{1}
\end{array}\right]
$$

and therefore

$$
G_{1} B K=\left[\begin{array}{l}
G_{11}^{1} K \\
G_{21}^{1} K
\end{array}\right] \text {. }
$$

By choosing the matrix $G_{11}^{1}$ invertible (and symmetric), there always exists a matrix $S \in \mathbb{R}^{(n-m) \times m}$ so that

$$
G_{21}^{1}=S G_{11}^{1} .
$$


By the change of variables $\hat{K}=G_{11}^{1} K$, the matrix $\Omega_{1}$ can be rewritten under the following form:

$$
\Omega_{1}=\left[\begin{array}{cc}
P-G-G^{T} \\
(\star)
\end{array}\left[\begin{array}{cc}
G_{1} A-\left[\begin{array}{c}
\hat{K} \\
S \hat{K}
\end{array}\right] & {\left[\begin{array}{c}
\hat{K} \\
S \hat{K}
\end{array}\right]} \\
0 & G_{2} A-\hat{L} C
\end{array}\right] .\right.
$$

The matrix inequality (22), when taking into account the new expression of $\Omega_{1}$ in (27), is not linear because of the presence of the coupling term $S \hat{K}$. To linearize it, we proceed as follows:

- Step 1 Solve the optimization problem resulting from the stabilization of (1a) by a static state feedback $u=$ $-\mathcal{K} x$ with respect the decision variables $\mathcal{P}, \mathcal{K}$ and a certain slack variable $\hat{G}=\left[\begin{array}{ll}\hat{G}_{11} & \hat{G}_{12} \\ \hat{G}_{12}^{T} & \hat{G}_{22} \cdot\end{array}\right]$ comming from Theorem 3 , and compute the matrix $\bar{S}:=\hat{G}_{11} \hat{G}_{12}$

- Step 2 Solve the optimisation problem

$$
\operatorname{minimize}\left(\hat{\alpha}+\hat{\beta}+\epsilon_{1}+\epsilon_{2}\right) \text { subject to 22 }-27 \text {. }
$$

with $S=\alpha \bar{S}$ by using the gridding method on $\alpha$ with the decision variables $P, \hat{K}, \hat{L}$.

We consider the stabilization problem of (1) by a static state feedback $u=-\mathcal{K} x$. System (1a) can be written in the closed-loop form:

$$
x_{t+1}=\left(A-B \mathcal{K}+M_{1} F_{1}(t) N_{1}\right) x_{t} .
$$

System (29) is globally asymptotically stable by the Lyapunov function $V(x)=x^{T} \mathcal{P} x$ if the following inequality holds:

$$
-\mathcal{P}+(A-B \mathcal{K}+\Delta A)^{T} \mathcal{P}(\mathcal{A}-B \mathcal{K}+\Delta A)<0
$$

Inequality 30 is equivalent, thanks to Theorem 3, to the existence of an invertible matrix $\mathcal{G}$ such that

$$
\left[\begin{array}{cc}
\mathcal{P}-\mathcal{G}-\mathcal{G}^{T} & \mathcal{G}(A-B \mathcal{K}+\Delta A) \\
\star & -\mathcal{P}
\end{array}\right]<0 .
$$

By choosing $\mathcal{G}=\mathcal{G}^{T}$, and using the congruence principal, we obtain that 30 holds if the following one is fulfilled:

$$
\operatorname{diag}\left(\mathcal{G}^{-1}, \mathcal{G}^{-1}\right) \mathfrak{F} \operatorname{diag}\left(\mathcal{G}^{-1}, \mathcal{G}^{-1}\right)<0,
$$

where

$$
\mathfrak{F}=\left[\begin{array}{cc}
\mathcal{P}-\mathcal{G}-\mathcal{G}^{T} & \mathcal{G}(A-B \mathcal{K}+\Delta A) \\
\star & -\mathcal{P}
\end{array}\right] .
$$

Thus, after developing and by making the changes of variables $\mathcal{Z}=\mathcal{G}^{-1} \mathcal{P G}^{-1}, \mathcal{G}^{-1}=\hat{G}$ and $\overline{\mathcal{K}}=\mathcal{K} \hat{G}$, we get 31 is

$$
\begin{aligned}
& {\left[\begin{array}{cc}
\mathcal{Z}-\hat{G}-\hat{G}^{T} & A \hat{G}-B \overline{\mathcal{K}} \\
\star & -\mathcal{Z}
\end{array}\right]} \\
& \quad+\operatorname{He}\left(\left[\begin{array}{c}
M_{A} \\
0
\end{array}\right] F_{A}(t)\left[\begin{array}{c}
0 \\
\left(N_{A} \hat{G}\right)^{T}
\end{array}\right]^{T}\right)<0 .
\end{aligned}
$$

Inequalities (33) can be easily linearized thanks to Young's inequality. Now, using the change of variable: $\hat{\alpha}_{S}=\alpha^{-2} \eta^{-1}$, where $\eta$ is a positive number that results from the use of Young's inequality. We deduce that (29) is globally asymptotically stable if the following LMI condition is fulfilled:

$$
\left[\begin{array}{cccc}
\mathcal{Z}-\hat{G}-\hat{G}^{T} & A \hat{G}-B \overline{\mathcal{K}} & M_{A} & 0 \\
(\star) & -\mathcal{Z} & 0 & \hat{G} N_{A}^{T} \\
(\star) & (\star) & -\eta I & 0 \\
(\star) & (\star) & (\star) & -\hat{\alpha}_{S} I
\end{array}\right]<0,
$$

Since our objective consists in optimizing the bounds of the uncertainties, so under this consideration we must solve the following convex optimization problem:

$$
\min \left(\hat{\alpha}_{S}+\eta\right) \text { subject to 34. }
$$

Our approach is reported and summarized in the following modified two-steps algorithm, that we call "MTSM":

\section{Algorithm MTSM:}

1) Solve the optimization problem: minimize $\left(\hat{\alpha}_{S}+\eta\right)$ subject to LMI (34) with the decision variables $\mathcal{Z}, \mathcal{K}$, $\hat{G}, \eta>0$, and $\hat{\alpha}_{S}$ and go to 2$)$;

2) Compute $\bar{S}$ by $\bar{S}:=\hat{G}_{21}\left(\hat{G}_{11}\right)^{-1}$, put $S=\bar{S} \Theta$, with $\Theta=\operatorname{diag}\left(\alpha_{1}, \alpha_{2}, \ldots, \alpha_{m}\right)$ and go to 3$)$;

3) Solve the optimization problem: $\operatorname{minimize}\left(\hat{\alpha}+\hat{\beta}+\epsilon_{1}+\epsilon_{2}\right)$ subject to 22 -27 with $S=\bar{S} \Theta$ by using the gridding method on $\alpha_{i}, i=$ $1, \ldots, m$, with the decision variables $P, G_{2}, G_{1}, \hat{K}$, $\hat{L}, \epsilon_{1}, \epsilon_{2}, \hat{\alpha}$, and $\hat{\beta}$ and go to 4$)$;

4) Compute the observer-based controller gains as $K=$ $\left(\hat{G}_{11}\right)^{-1} \hat{K}, L=G_{2}^{-1} \hat{L}$.

Remark 2: In item 2), one could also compute the matrix $\bar{S}$ by $\bar{S}:=\mathcal{G}_{21} \mathcal{G}_{11}^{-1}$, since $\mathcal{G}$ and $\hat{G}$ are congruent matrices.

Before proceeding with the next section, we briefly comment what has been presented so far to clarify the novel contribution given by Algorithm 1 as compared with the results available in the current literature. In particular, a comparison is deserved with the approach proposed by Zemouche et al. [11] and that proposed by Stankovic et al. [12].

The existing two-steps method are different from our new design technique. Indeed, the standard method proceeds as in the following algorithm:

\section{Algorithm 1: (classical two-steps method (CTSM))}

1) Solve LMI (34) (with $P=G$ ), with the decision variables $\mathcal{Z}, \mathcal{K}$ and $\eta>0$

2) compute $\mathcal{K}$ by $\mathcal{K}=\overline{\mathcal{K}} \mathcal{Z}^{-1}$;

3) solve 22 for $K=\mathcal{K}$ with the decision variables $P_{1}, P_{2}, \hat{L}$ and $\epsilon_{i}, i=1,2$;

4) compute the observer gain as $L=P_{2}^{-1} \hat{L}$.

As can be seen, the CTSM algorithm calculates the gains $K$ and $L$ separately. 
Algorithm 2 (NTSM)

1) Solve LMI (34) with the decision variables $\mathcal{Z}, \overline{\mathcal{K}}$ and $\eta>0$

2) compute $\bar{S}$ by $\bar{S}=\left(\mathcal{P}_{12}\right)^{T} \mathcal{P}_{11}^{-1}$, and put $S=\alpha \bar{S}$;

3) solve 221-27) with $S=\bar{S} \Theta$, with $\Theta=$ $\operatorname{diag}\left(\alpha_{1}, \alpha_{2}, \ldots, \alpha_{m}\right)$, by using the gridding method on $\alpha_{i}$ with the decision variables $P_{1}, P_{2}, \hat{K}, \hat{L}$ and $\epsilon_{i}, i=1,2$;

4) compute the observer-based controller gains as $K=$ $P_{11}^{-1} \hat{K}$ and $L=P_{2}^{-1} \hat{L}$.

As said before, the introduction of the slack variable $G$ (in both step one and step two) in our algorithm renders it more general than NTSM algorithm. Indeed, if we take $\mathcal{G}=\mathcal{Z}$ in item 1) and $G=P$ in item 4) in our algorithm, we get exctely Algorithm 2 (NTSM). The advatage of our method lies in the semoultanious search for the decision variable $K, L$, and $P$ in the feasibility domain of the main LMI. Contrarely to Algorithm 2, where the research of Lyapunov matrix $P$ (which is a main decision variable) is done by "collage".

Remark 3: Other variants of the two-steps method can be derived. Indeed:

1) In the classical two step method, instead of solving (22) for $K=\mathcal{K}$, we can also consider $K=\overline{\mathcal{K}}$, or a more general structure by weighting $\overline{\mathcal{K}}$ by a slack variable $\Theta=\operatorname{diag}\left(\alpha_{1}, \alpha_{2}, \ldots, \alpha_{m}\right)$, namely, $K=\Theta \overline{\mathcal{K}}$, and then solve (22) by the gredding method with respect to $\alpha_{i}, i=1, \ldots, m$.

2) In the stability analysis, instead of considering the closed loop system provided by $z_{t}=\left(x_{t}^{T}, e_{t}^{T}\right)^{T}$, we can also consider that provided by $z_{t}=\left(\hat{x}_{t}^{T}, e_{t}^{T}\right)^{T}$. In this case, the first step is dedicated to the search of the gain matrice $L$, instead of $K$.

3) In Algorithm 2, we can propose two other possibilities. The first one requires no condition on the matrix $B$. It consists in evaluating (entirely) the Lyapunov matrix $P$ from the first step, by remplacing $P$ by $\mathcal{Z}$ (or by $\Theta \mathcal{Z}$ ), and then compute simultaniously the gains $K$ and $L$ from the second steps. The second one consists in remplacing $P$ by $\mathcal{P}$, or by $\Theta \mathcal{P}$.

All these senarios have the a common point, whose principle is to linearize the couplet term $P B K$ or $G_{1} B K$ with respect to: either $P$, or $K$, or $G_{1}$ from the first step. The idea of calculating a part of $P$ or $G$ is specific to the form of the matrix $B$, that must be a full rank matrix. In this case, we do not need to calculate a part of $P$ or $G_{1}$ of the first step. Mathematically, we do not yet know how to justify exactely these different choices. For example, choosing $G_{1}$ from the first step could be due to the fact that the variable $G_{1}$ (or part of $G_{1}$ ) must be in the stability domain of the closed loop system by static feedback, which implies that the system is stable when $L=0$ (this is the case, since the pair $(A, B)$ is assumed to be stabilizable.

\section{NUMERICAL EXAMPLE}

In this section, a numerical example is presented to illustrate the effectiveness of the proposed approach. We reconsider the example of [3] and [7], described by the following parameters:

$$
\begin{gathered}
A=\left[\begin{array}{ccc}
1 & 0.1 & 0.4 \\
1 & 1 & 0.5 \\
-0.3 & 0 & 1
\end{array}\right], B=\left[\begin{array}{cc}
0.1 & 0.3 \\
-0.4 & 0.5 \\
0.6 & 0.4
\end{array}\right], C=\left[\begin{array}{lll}
1 & 1 & 1 \\
1 & 1 & 1
\end{array}\right], \\
M_{A}=\gamma_{1}\left[\begin{array}{ccc}
0 & 0 & 0 \\
0.1 & 0.3 & 0.1 \\
0 & 0.2 & 0
\end{array}\right], N_{A}=\left[\begin{array}{ccc}
0 & 0 & 0 \\
0.2 & 0 & 0.4 \\
0 & 0.1 & 0
\end{array}\right] \\
M_{C}=\gamma_{2}\left[\begin{array}{ccc}
0 & 0 & 0.3 \\
0 & 0 & 0.8
\end{array}\right], N_{C}=\left[\begin{array}{ccc}
0 & 0 & 0 \\
0 & 0 & 0 \\
0 & 0 & 0.2
\end{array}\right] .
\end{gathered}
$$
By applying the transformation $T=\left[\begin{array}{ccc}\frac{50}{17} & -\frac{30}{17} & 0 \\ \frac{40}{17} & \frac{10}{17} & 0 \\ \frac{230}{119} & -\frac{10}{17} & -\frac{10}{14}\end{array}\right]$,
on the previous system, the following new parameters $A, B$, $C, M_{A}, N_{A}, M_{C}, N_{C}$ are reached:

$$
\begin{gathered}
A:=\left[\begin{array}{ccc}
0.8824 & -0.2647 & -0.4118 \\
0.7059 & 1.7882 & -1.7294 \\
0.1727 & 0.1761 & 0.3294
\end{array}\right], B:=\left[\begin{array}{ll}
1 & 0 \\
0 & 1 \\
0 & 0
\end{array}\right], \\
C:=\left[\begin{array}{lll}
0.3 & 1.2 & -1.4 \\
0.3 & 1.2 & -1.4
\end{array}\right], M_{C}:=\gamma_{2}\left[\begin{array}{lll}
0 & 0 & 0.3 \\
0 & 0 & 0.8
\end{array}\right], \\
M_{A}:=\gamma_{1}\left[\begin{array}{ccc}
-0.1765 & -0.5294 & -0.1765 \\
-0.0588 & 0.1765 & 0.0588 \\
-0.0588 & -0.3193 & -0.0588
\end{array}\right], \\
N_{A}=\left[\begin{array}{ccc}
0 & 0 & 0 \\
0.2600 & 0.2200 & -0.5600 \\
-0.0400 & 0.0500 & 0
\end{array}\right], \\
N_{C}:=\left[\begin{array}{ccc}
0 & 0 & 0 \\
0 & 0 & 0 \\
0.1200 & 0.0800 & -0.2800
\end{array}\right] .
\end{gathered}
$$

By choosing $\gamma_{1}=\gamma_{2}=1$, we solved the LMI (22)-(27) of Algorithm, we obtain the matrices gains

$K^{T}=\left[\begin{array}{cc}1.0311 & 0.7324 \\ 0.0544 & 1.5474 \\ -0.5672 & -1.8033\end{array}\right], L=\left[\begin{array}{cc}-1.7623 & 0.6609 \\ 1.5539 & -0.5827 \\ -0.9256 & 0.3471\end{array}\right]$.

Now, we searched for the maximum values of $\gamma_{1}$ and $\gamma_{2}$ that satisfy the above described LMI. After solving the optimization problem (22)-27), we get $\gamma_{1 \max }=5.78, \gamma_{2 \max }=$ $10^{15}$. The results are summarized in Table I]

\begin{tabular}{|c||c|c|c|c|c|}
\hline \hline LMIs & $\begin{array}{c}\text { LMIs [10]-(12) } \\
{[3]}\end{array}$ & $\begin{array}{c}\text { LMI [9] } \\
{[7]}\end{array}$ & MTSM & $\begin{array}{c}\text { NTSM } \\
{[11]}\end{array}$ & $\begin{array}{c}\text { CTSM } \\
{[12]}\end{array}$ \\
\hline$\gamma_{1 \max }$ & 2.89 & 4.64 & 5.78 & 5.5 & 5.08 \\
\hline$\gamma_{2 \max }$ & $10^{12}$ & $10^{13}$ & $10^{15}$ & $10^{15}$ & $10^{15}$ \\
\hline \hline
\end{tabular}

TABLE I

COMPARISON BETWEEN DIFFERENT LMI DESIGN METHODS

The obtained matrices gains are:

$$
K^{T}=\left[\begin{array}{cc}
0.1491 & 0.8117 \\
-0.5840 & 1.6815 \\
-0.1615 & -2.3640
\end{array}\right], L=\left[\begin{array}{cc}
-1.7788 & 0.66671 \\
1.9689 & -0.7383 \\
-0.9227 & 0.3460
\end{array}\right]
$$

The simulation results corresponding to the observer-based controller gains are given in Figure (1) and (2) with $x_{0}=$ $\left[\begin{array}{lll}-1 & 2 & 5\end{array}\right]^{T}$ and $\hat{x}_{0}=\left[\begin{array}{lll}1 & 5 & -7\end{array}\right]^{T}$. 

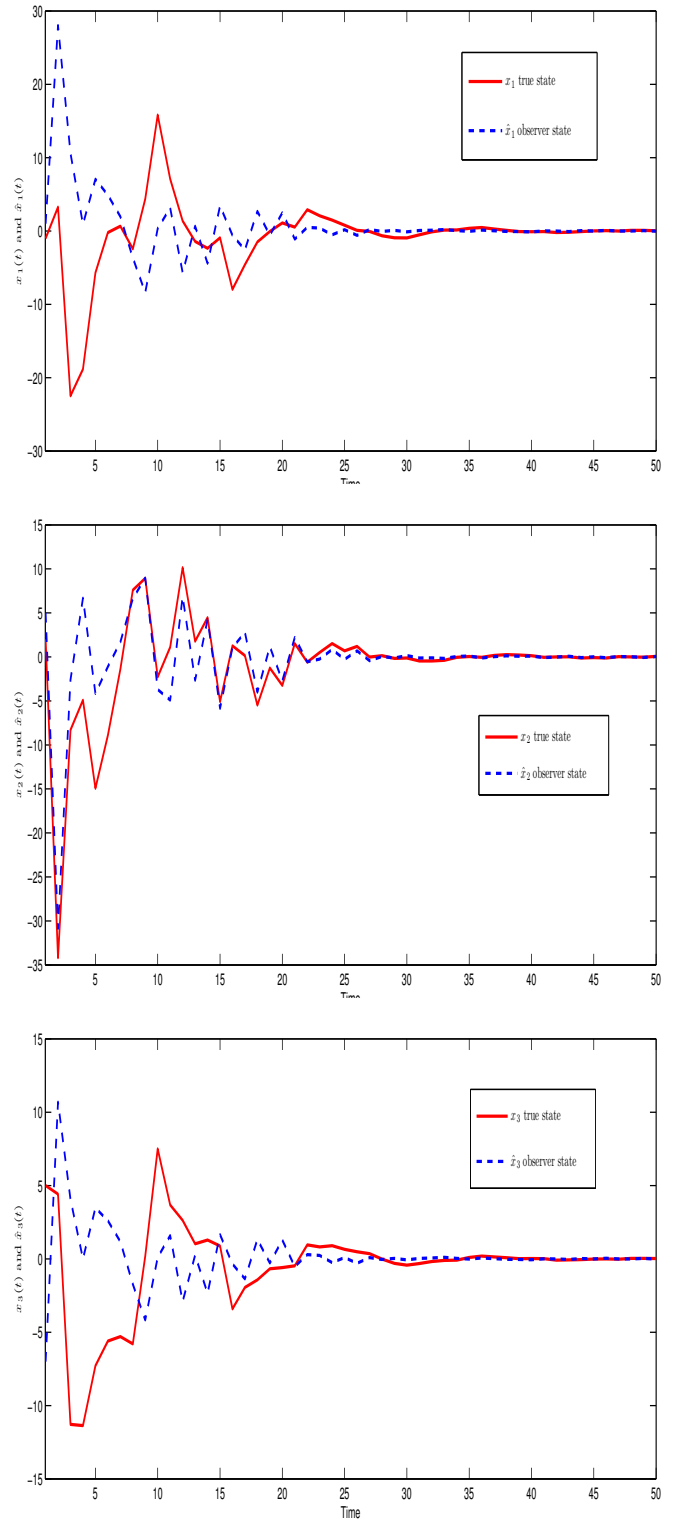

Fig. 1. Behavior of $x$ and $\hat{x}$

\section{CONCLUSION}

In this paper, a new variant of two-steps algorithm to construct observer-based controllers for uncertain linear discretetime is proposed via the satisfaction of a two LMIs. The connection with respect to existing two-steps algorithms in the literature are analyzed also via numerical tests, which show an increased feasibility as compared with alternative design methods. Future work will concern possible extensions of the proposed approach to the design of observerbased controllers for nonlinear delay systems.

\section{REFERENCES}

[1] C. Lien, "Robust observer-based control of systems with state perturbations via LMI approach," IEEE Transactions on Automatic Control, vol. 49, no. 8, pp. 1365-1370, 2004.

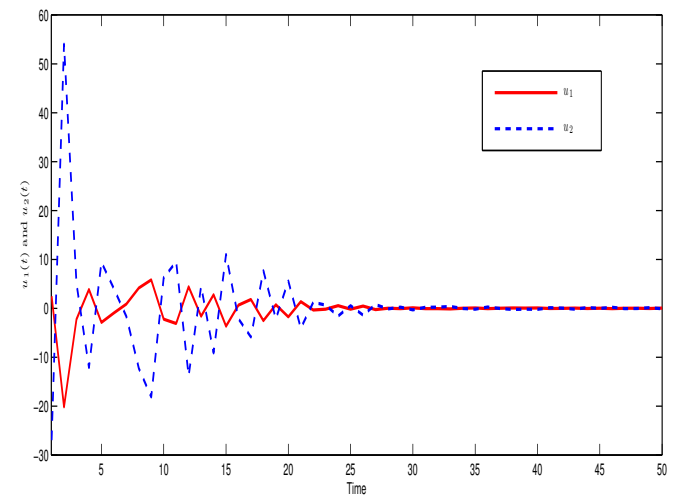

Fig. 2. Behavior of $u_{1}$ and $u_{2}$

[2] S. Ibrir, W. Xie, and C. Su, "Observer-based control of discrete-time Lipschitzian non-linear systems: application to one-link flexible joint robot," International Journal of Control, vol. 78, no. 6, pp. 385-395, 2005.

[3] S. Ibrir and S. Diopt, "Novel LMI conditions for observer-based stabilization of Lipschitzian nonlinear systems and uncertain linear systems in discrete-time," Applied Mathematics and Computation, vol. 206, no. 2, pp. 579-588, 2008.

[4] S. Ibrir, "Static output feedback and guaranteed cost control of a class of discrete-time nonlinear systems with partial state measurements,' Nonlinear Analysis, vol. 68, no. 7, pp. 1784-1792, 2008.

[5] — "Design of static and dynamic output feedback controllers through Euler approximate models: uncertain systems with normbounded uncertainties," IMA J. Math. Control Inform., vol. 25, no. 3, pp. 281-296, 2008.

[6] H. Kheloufi, A. Zemouche, F. Bedouhene, and M. Boutayeb, "On LMI conditions to design observer-based controllers for linear systems with parameter uncertainties," Automatica, vol. 49, no. 12, pp. 3700-3704, 2013.

[7] H. Kheloufi, F. Bedouhene, A. Zemouche, and A. Alessandri, "Observer-based stabilisation of linear systems with parameter uncertainties by using enhanced LMI conditions," International Journal of Control, vol. 88, no. 6, pp. 1189-1200, 2015.

[8] B. Grandvallet, A. Zemouche, H. Souley-Ali, and M. Boutayeb, "New lmi condition for observer-based $\mathcal{H}_{\infty}$ stabilization of a class of nonlinear discrete-time systems," SIAM Journal on Control and Optimization, vol. 51, no. 1, pp. 784-800, 2013.

[9] W. Heemels, J. Daafouz, and G. Millerioux, "Observer-based control of discrete-time LPV systems with uncertain parameters," IEEE Transactions on Automatic Control,, vol. 55, no. 9, pp. 2130-2135, 2010.

[10] L. Jetto and V. Orsini, "Efficient LMI-based quadratic stabilization of interval LPV systems with noisy parameter measures," IEEE Transactions on Automatic Control, vol. 55, no. 4, pp. 993 -998, 2010.

[11] A. Zemouche, R. Rajamani, H. Kheloufi, and F. Bedouhene, "Robust observer-based stabilization of lipschitz nonlinear uncertain systems via lmis - discussions and new design procedure," International Journal of Robust and Nonlinear Control, pp. n/a-n/a, 2016, rnc.3644. [Online]. Available: http://dx.doi.org/10.1002/rnc.3644

[12] S. Stankovic, D. Stipanovic, and D. Siljak, "Decentralized dynamic output feedback for robust stabilization of a class of nonlinear interconnected systems," Automatica, vol. 43, no. 5, pp. 861-867, 2007.

[13] M. de Oliveira, J. Bernussou, and J. Geromel, "A new discrete-time robust stability condition,” Systems \& Control Letters, vol. 37, no. 4, pp. $261-265,1999$. 